\title{
Análise de Algumas Variáveis Fisiológicas para Avaliação do Grau de Adaptação de Ovinos Submetidos ao Estresse por Calor ${ }^{1}$
}

\author{
Josiane Maria Cardoso Starling ${ }^{2}$, Roberto Gomes da Silva ${ }^{3}$, Mario Cerón-Muñoz ${ }^{4}$, \\ Geraldo Sérgio Senra Carneiro Barbosa ${ }^{5}$, Mateus José Rodrigues Paranhos da Costa ${ }^{3}$
}

\begin{abstract}
RESUMO - Investigaram-se a temperatura retal, a freqüência respiratória e a taxa de evaporação total de ovinos Corriedale sob três temperaturas ambientes, visando uma melhor compreensão dos mecanismos de termorregulação desses animais. Inicialmente, 21 animais adultos foram alojados em câmara climática à temperatura de $45^{\circ} \mathrm{C}$, e pressão parcial de vapor (PV) variável, registrando-se a freqüência respiratória (FR) e a temperatura retal (TR). Baseando-se na FR e TR, foram selecionados 10 animais, cinco com os valores mais baixos, assumindo-os como mais adaptados ao calor (grupo 1) e cinco com valores mais altos, assumindo-os como menos adaptados (grupo 2). Os animais selecionados foram mantidos em câmara climática, onde mediram-se novamente TR, FR e taxa de evaporacão total (TET), sob 20,30 e $40^{\circ} \mathrm{C}$ de temperatura do ar e PV variável. Não houve diferença estatística entre os grupos classificados, para todas as variáveis medidas. Concluiu-se que a utilização das variáveis fisiológicas TR e FR como parâmetros únicos para a seleção destes animais não é suficiente para avaliar o grau de adaptação a temperaturas elevadas.
\end{abstract}

Palavras-chave: estresse por calor, ovinos, termólise evaporativa

\section{Analysis of Some Physiological Variables for the Evaluation of the Degree of Adaptation in Sheep Submitted to Heat Stress}

\begin{abstract}
It was investigated the rectal temperature, respiratory frequency and total evaporative heat loss rate in Corriedale sheep under three air temperatures, aiming a better comprehension of thermoregulation mechanisms of these animals. Initially, 21 adult animals were housed in climatic chamber under $45^{\circ} \mathrm{C}$ and variable air humidity (PV), recording the respiratory frequency (FR) and rectal temperature (TR). Basing on the FR and TR, it was selected 10 animals, five of the lowest values, assuming as being the best heat adapted (group 1) and five of the highest values, assuming as the worst heat adapted (group 2). The selected animals were maintained in climatic chamber, where it was measured again TR, FR and total evaporation rate (TET), under 20,30 and $40^{\circ} \mathrm{C}$ of air temperature and variable PV. There was no statistical difference between the classified groups, for all the measured variables. In conclusion, the use of the physiological variables TR and FR as mainly parameters for these animals selection, is not enough for evaluate the level of adaptation under high temperatures.
\end{abstract}

Key Words: heat stress, evaporative heat loss, sheep

\section{Introdução}

Geralmente, em um ambiente tropical, o mecanismo físico de termólise considerado mais eficaz é o evaporativo, por não depender do diferencial de temperatura entre o organismo e a atmosfera. Nesses ambientes, a temperatura do ar tende a ser próxima ou maior que a corporal, tornando ineficazes as termólises por condução e convecção (Silva, 2000). Portanto, a evaporação no trato respiratório ou na superfície da pele é um mecanismo essencial para a regulação térmica em homeotérmicos (Cena \& Monteith, 1975).
Em ambiente de temperatura muito elevada, tanto o excesso como a carência de umidade serão prejudiciais. Se o ambiente é quente e muito seco a evaporação é rápida, podendo causar irritação cutânea e desidratação geral; no caso do ambiente ser quente e demasiadamente úmido, a evaporação torna-se muito lenta ou nula, reduzindo a termólise e aumentando a carga de calor do animal, principalmente porque, em condições de alta temperatura, a termólise por convecção é prejudicada.

A quantidade de água evaporada do corpo de um animal depende de diversos fatores, de acordo com o

\footnotetext{
${ }_{1}^{1}$ Parte da tese de Doutorado da primeira autora, apresentada à FCAV - UNESP, Jaboticabal/SP, financiada pela FAPESP

2 Prof. Dra. da Faculdade de Medicina Veterinária da UNIFRAN, Franca/SP - Cx Postal 113 - Jaboticabal/SP - cep.: $14.870-970$.

E.mail: starling@fcav.unesp.br

3 Prof. Dr. do Departamento de Zootecnia - FCAV - UNESP, Jaboticabal/SP. E.mail: robertogomes@netsite.com.br; mpcosta@fcav.unesp.br

${ }^{4}$ Doutorando do curso de Produção Animal, Zootecnia - FCAV - UNESP, Jaboticabal/SP.

5 Prof. do Departamento de Zootecnia - CEDAF - UFV, Florestal/ MG. E.mail: gsenra@ufv.br
} 
local em que ocorre a evaporação. No caso desta ocorrer na superfície do aparelho respiratório, são fatores importantes: o volume de ar respirado, a temperatura corporal e a umidade do ar inspirado. Para a evaporação na superfície da epiderme, os fatores mais relevantes são: a velocidade do vento, a temperatura ambiente, a umidade do ar, a taxa de transferência de água para a superfície cutânea, as características da capa de cobertura (pelame, velo) e a temperatura da superfície. Por sua vez, alguns desses fatores podem associar-se a outros, como idade, sexo, raça, ambiente radiante, etc (Silva, 2000).

A intensidade da radiação solar está relacionada com a temperatura do ambiente onde o animal vive e influencia os tecidos que revestem seu corpo. A radiação solar direta, nas faixas ultravioleta, luz visível e infravermelha, é, em parte, refletida de acordo com a cor e outras propriedades do pelame do animal, sendo a parte restante, absorvida sob a forma de calor. Desta forma, a capa externa do organismo, constituída pelo pelame ou velo nos mamíferos e penas e penugem nas aves, assume fundamental importância para as trocas térmicas entre o organismo e o ambiente. Nas regiões tropicais, a capa externa possui funções mais relacionadas à proteção mecânica da epiderme, ao mimetismo e à proteção contra a radiação solar (Silva, 2000). A distinta disposição isolante da superfície corporal (revestimento piloso, plumagem, tecido adiposo subcutâneo), assim como das glândulas sudoríparas, leva à existência de peculariedades na regulação do equilíbrio térmico, que dependem da espécie animal que apresentará diferentes sensibilidades ao aumento e decréscimo da temperatura ambiental (Kolb, 1976).

O total de água eliminada pelo organismo, por meio da evaporação, pode ser estimado por medição gravimétrica, que requer a utilização de uma balança de elevada sensibilidade. Assim, Holmes (1985) propôs um método prático envolvendo um sistema de pesagem móvel, desenvolvido originalmente para monitoramento de perdas de líquidos de pacientes humanos em hospitais. Finch (1986) utilizou esse sistema em experimentos com bovinos, obtendo sucesso nos resultados, inclusive em condições de campo. Posteriormente, Silva \& Gaudiosi (1995), ao testarem o método gravimétrico com ovinos em câmara climática (temperatura: 25 a $45^{\circ} \mathrm{C}$, pressão parcial de vapor: 1,3 a 4,5 $\mathrm{KPa}$ ), verificaram que o principal fator de variação foi a frequiência respiratória, em uma relação direta com a taxa de evaporação total e que a termólise evaporativa em ovinos lanados processa-se, principalmente, por meio das vias respiratórias, caracterizando, assim, a eficiente proteção do velo contra as perdas evaporativas cutâneas. Esse fato também foi observado por Brockway et al. (1965) e Cena \& Monteith (1975).

Assim, o presente trabalho foi conduzido com o objetivo de testar a hipótese de que o grau de adaptação de ovinos ao calor pode ser avaliado por intermédio de medidas da temperatura retal e frequiência respiratória, que seriam positivamente associadas à taxa de evaporação total.

\section{Material e Métodos}

O presente trabalho foi conduzido nas instalações do Laboratório de Bioclimatologia Animal, Departamento de Zootecnia, da Faculdade de Ciências Agrárias e Veterinárias da Universidade Estadual Paulista (UNESP), Campus de Jaboticabal/SP $\left(21^{\circ} 15^{\prime} 22^{\prime \prime}\right.$ de Latitude Sul, $48^{\circ} 18^{\prime} 58^{\prime \prime}$ Longitude Oeste e 595 m de altitude), e dividido em duas fases.

$\mathrm{Na}$ fase I, foram utilizados 21 ovinos da raça Corriedale (7 fêmeas e 14 machos) com aproximadamente um ano de idade. As fêmeas estavam vazias e não lactantes. Nessa fase, objetivou-se selecionar, por meio da avaliação da temperatura retal e da frequiência respiratória, dois grupos de animais, classificando-os em mais adaptados (grupo 1) e menos adaptados (grupo 2) ao estresse pelo calor, quando submetidos à temperatura do ar de $45^{\circ} \mathrm{C}$, conforme descrito adiante.

Os animais foram alojados em uma câmara climática, com $36 \mathrm{~m}^{2}$ de área (6 x $6 \mathrm{~m}$ ) e $3 \mathrm{~m}$ de altura, sendo a iluminação interior de luz fluorescente. No centro da câmara, foram instaladas quatro baias com $10,4 \mathrm{~m}^{2}$ de área total, com piso ripado a $25 \mathrm{~cm}$ de altura do solo. Cada baia possuía dois cochos suspensos e um balde de plástico como bebedouro, com capacidade de 18 litros. Para o aquecimento do ar no interior da câmara, foram utilizados seis radiadores da marca Arno com $1200 \mathrm{~W}$ de potência cada um, além de um aquecedor central que lançava ar quente no seu interior. A circulação do ar era proporcionada por um exaustor junto ao solo, que retirava o ar menos quente do piso da câmara, passando por uma sala lateral à câmara climática e retornando pelo interior do aquecedor central.

Ao centro da câmara climática foi instalado um psicrômetro (de bulbo seco e bulbo úmido), utilizado 
para o monitoramento da temperatura e da umidade do ar. A umidade do ar foi expressa como pressão parcial de vapor $(\mathrm{Pp})$, calculada a partir dos registros de temperatura do bulbo úmido (tu) e do bulbo seco (ta), segundo a fórmula:

$$
P_{p}\left\{t_{a}\right\}=P_{s}\left\{t_{u}\right\}-\gamma\left(t_{a}-t_{u}\right), k P a,
$$

em que: $\mathrm{P}_{\mathrm{s}}\left\{\mathrm{t}_{\mathrm{u}}\right\}=$ pressão de saturação à temperatura $\mathrm{t}_{\mathrm{u}}=0,61078 \times 10(7,5 \mathrm{tu} /(\mathrm{tu}+237,5) ; \gamma=$ constante psicrométrica para a temperatura ta, obtida em tabela de características do ar; $\mathrm{t}_{\mathrm{a}}=$ temperatura de bulbo seco, ${ }^{\circ} \mathrm{C} ; \mathrm{t}_{\mathrm{u}}=$ temperatura de bulbo úmido, ${ }^{\circ} \mathrm{C}$.

Os animais eram alojados diariamente na câmara climática das 8 às $16 \mathrm{~h}$, durante sete dias consecutivos. Após as oito horas diárias de monitoramento, os animais eram transferidos para um piquete adjacente à câmara climática. A temperatura do ar na câmara era controlada para manter-se a $45^{\circ} \mathrm{C}$, sendo a umidade do ar variável. Os dados eram registrados três vezes ao dia (9, 14 e $16 \mathrm{~h})$.

A frequiência respiratória foi medida por meio da contagem dos movimentos respiratórios no flanco dos animais, com o auxílio de um cronômetro; a temperatura retal, por meio de um termômetro clínico.

Durante o período de permanência dos animais no interior da câmara, os mesmos recebiam como alimento feno de coastcross picado (Cynodon dactylon) e concentrado comercial, misturados na proporção 40:60, respectivamente, com fornecimento ad libtum nos cochos instalados nas baias. A água também era oferecida à vontade.

A partir dos dados colhidos, foi realizada análise estatística pelo método dos quadrados mínimos, segundo o procedimento GLM do pacote computacional SAS (1996), utilizando-se o seguinte modelo matemático:

$$
\mathrm{Y}_{\mathrm{ijkl}}=\mu+\mathrm{d}_{\mathrm{i}}+\mathrm{a}_{\mathrm{ij}}+\mathrm{h}_{\mathrm{k}}+\mathrm{e}_{\mathrm{ijkl}}
$$

em que: $Y_{\mathrm{ijkl}}=$ temperatura retal ou freqüência respiratória do j-ésimo animal, no k-ésimo horário, no i-ésimo dia; $\mu=$ média geral; $\mathrm{d}_{\mathrm{i}}=$ efeito fixo do i-ésimo dia $(i=1, \ldots, 14) ; a_{i j}=$ efeito fixo do j-ésimo animal dentro do i-ésmo dia $(j=1, \ldots, 21) ; h_{k}=$ efeito fixo do k-ésimo horário $(\mathrm{k}=1,2,3)$; $\mathrm{e}_{\mathrm{ijkl}}=$ resíduo.

Com os resultados obtidos utilizando-se o modelo acima, foi feita a análise de componentes principais e constituiu-se um índice para cada animal, combinando as informações de temperatura retal e freqüência respiratória (ITF). Este índice de adaptação ao calor foi obtido a partir do autovetor estimado para o maior autovalor, da matriz de variâncias e covariâncias. Em seguida, o ITF foi aplicado a todos os animais, que foram ordenados segundo o valor final obtido.

A partir desta análise, selecionou-se 10 animais (sete machos e três fêmeas), constituindo dois grupos que foram utilizados posteriormente na fase II, realizando-se as medições da taxa de evaporação total. $\mathrm{O}$ grupo 1 foi formado pelos cinco indivíduos considerados mais adaptados às altas temperaturas com menor reação ao estresse pelo calor, com manutenção dentro dos limites normais da frequiência respiratória e da temperatura retal, correspondentes aos menores valores do ITF. O grupo 2 foi formado pelos cinco indivíduos com reação mais intensa ao estresse pelo calor, com aumento da freqüência respiratória e maior desvio da temperatura retal normal, correspondentes aos maiores valores do ITF.

$\mathrm{Na}$ fase II, os animais foram mantidos na mesma câmara climática para as medições da termólise evaporativa total, que foram realizadas no período de 03 de agosto a 02 de outubro de 1998. Após três anos da última tosquia realizada nos animais, as fêmeas apresentavam variação de 7,5 a $8,1 \mathrm{~cm}$ e os machos 9,7 a $13,0 \mathrm{~cm}$ na espessura do velo.

Cada um dos 10 animais do lote era alojado em uma gaiola metálica, do tipo usado em estudos de digestibilidade, cujo piso era de chapa perfurada, de modo a recolher os dejetos dos animais em um recipiente com óleo, para prevenir a evaporação da umidade dos dejetos e assim evitar alterações indevidas no peso. A gaiola era instalada na câmara climática, onde cada animal permanecia por duas horas, sem receber água ou alimento.

Todos os animais foram observados sob três faixas diferentes de temperatura do ar e pressão parcial de vapor variando de 1,53 a 3,23 KPa (média de 2,36 $\pm 0,46 \mathrm{KPa}$ ), por três semanas consecutivas para cada temperatura, inicialmente à temperatura do ar de $20^{\circ} \mathrm{C}$, em seguida a $30^{\circ} \mathrm{C}$ e, posteriormente, a $40^{\circ} \mathrm{C}$. Conseqüentemente, foram obtidas três repetições para cada animal.

Foi utilizado o sistema de pesagem de alta sensibilidade WM-204 (fabricado por Scale-Tronix, Inc., White Plains, New York, USA), desenvolvido originalmente para o monitoramento de perdas de líquidos em pacientes humanos, sendo o mesmo utilizado em animais por Holmes (1985), Finch (1986) e Silva \& Gaudiosi (1995). Basicamente, o sistema é constituído por quatro transdutores tipo strain-gauge, colocados sob o suporte da gaiola onde se aloja o animal e acoplados a uma unidade central computadorizada, 
que registra continuamente as alterações de peso com sensibilidade de $10 \mathrm{~g}$. A capacidade do sistema atinge cerca de $500 \mathrm{~kg}$.

A perda total de água eliminada por evaporação (cutânea e respiratória) era registrada de cinco em cinco minutos e referida ao peso do animal. Ao final da observação, media-se a temperatura retal e a frequiência respiratória. A diferença entre o peso corporal inicial e o final, atribuída ao total de água evaporada em cada sessão de 2 horas, era convertida em gramas de água por $\mathrm{kg}$ de peso corporal por hora $(\mathrm{g} / \mathrm{kg} / \mathrm{h})$.

Também foram registradas, no local, as temperaturas de bulbo seco e bulbo úmido antes e após as duas horas de observação.

O calor dissipado por evaporação $(\mathrm{Q})$ foi estimado para cada valor de temperatura do ar $(20,30$ e $40^{\circ} \mathrm{C}$ ), utilizando-se a seguinte equação, de acordo com Silva \& Gaudiosi (1995):

$$
\mathrm{Q}=\mathrm{Y} \lambda / 3600 ; \text { Watts/kg }
$$

em que: $\mathrm{Y}$ é a quantidade de água evaporada (ou taxa de evaporação), $\lambda=2501-2,372727 \mathrm{~T}$, é o calor latente de vaporização, estimado para a temperatura do ar (T).

Utilizou-se o seguinte modelo estatístico para a análise da taxa de evaporação total:

$$
\begin{gathered}
\mathrm{Y}_{\mathrm{ijklm}}=\mu+\mathrm{g}_{\mathrm{i}}+\mathrm{s}_{\mathrm{j}}+\mathrm{a}_{\mathrm{ijk}}+\mathrm{t}_{1}+\mathrm{b}_{1} \mathrm{p}_{1}+\mathrm{b}_{2} \mathrm{r}_{1} \\
+\mathrm{b}_{3} \mathrm{f}_{1}+\mathrm{e}_{\mathrm{ijklm}}
\end{gathered}
$$

em que: $\mathrm{Y}_{\mathrm{ijklm}}=\mathrm{m}$-ésima medida da taxa de evaporação total efetuada no k-ésimo animal do i-ésimo grupo, pertencente ao j-ésimo sexo, na l-ésima temperatura do ar; $\mu=$ média geral; $\mathrm{g}_{\mathrm{i}}=$ efeito do i-ésimo grupo $(i=1,2) ; s_{j}=$ efeito do $j$-ésimo sexo; $\mathrm{a}_{\mathrm{ijk}}=$ efeito do k-ésimo animal dentro do i-ésimo grupo e do j-ésimo sexo $(\mathrm{k}=1, \ldots, 10) ; \mathrm{t}_{1}=$ efeito da l-ésima temperatura do ar $(1=20,30,40) ; b_{1} p_{1}=$ regressão sobre a pressão parcial de vapor; $\mathrm{b}_{2} \mathrm{r}_{1}=$ regressão sobre a temperatura retal; $\mathrm{b}_{3} \mathrm{f}_{1}=$ regressão sobre $\mathrm{a}$ freqüiência respiratória; $\mathrm{e}_{\mathrm{ijklm}}=$ resíduo.

Para a análise da temperatura retal, foi utilizado o modelo:

$$
\begin{gathered}
\mathrm{Y}_{\mathrm{ijklm}}=\mu+\mathrm{g}_{\mathrm{i}}+\mathrm{s}_{\mathrm{j}}+\mathrm{a}_{\mathrm{ijk}}+\mathrm{t}_{1}+\mathrm{b}_{1} \mathrm{p}_{1}+\mathrm{b}_{2} \mathrm{r}_{1} \\
+\mathrm{b}_{3} \mathrm{f}_{1}+\mathrm{e}_{\mathrm{ijklm}}
\end{gathered}
$$

em que: $\mathrm{Y}_{\mathrm{ijklm}}=\mathrm{m}$-ésima medida da temperatura retal efetuada no k-ésimo animal do i-ésimo grupo, pertencente ao j-ésimo sexo, na l-ésima temperatura do ar; $\mu=$ média geral; $\mathrm{g}_{\mathrm{i}}=$ efeito do i-ésimo grupo $(\mathrm{i}=1,2) ; \mathrm{s}_{\mathrm{j}}=$ efeito do $\mathrm{j}$-ésimo sexo; $\mathrm{a}_{\mathrm{ijk}}=$ efeito do k-ésimo animal dentro do i-ésimo grupo e do j-ésimo sexo $(\mathrm{k}=1, \ldots, 10) ; \mathrm{t}_{1}=$ efeito da 1 -ésima temperatura do $\operatorname{ar}(1=20,30,40) ; b_{1} p_{1}=$ regressão sobre a pressão parcial de vapor; $\mathrm{b}_{2} \mathrm{r}_{1}=$ regressão sobre a taxa de evaporação total; $\mathrm{b}_{3} \mathrm{f}_{1}=$ regressão sobre a freqüência respiratória; $\mathrm{e}_{\mathrm{ijklm}}=$ resíduo.

Para a freqüência respiratória, o modelo estatístico foi:

$$
\begin{gathered}
Y_{i j k l m}=\mu+g_{i}+s_{j}+a_{i j k}+t_{1}+b_{1} p_{1}+b_{2} r_{1} \\
+b_{3} f_{1}+e_{i j k l m}
\end{gathered}
$$

em que: $Y_{i j k l m}=m$-ésima medida da frequiência respiratória efetuada no k-ésimo animal do i-ésimo grupo, pertencente ao j-ésimo sexo, na l-ésima temperatura do ar; $\mu=$ média geral; $g_{i}=$ efeito do i-ésimo grupo $(i=1,2) ; s_{j}=$ efeito do $j$-ésimo sexo; $a_{i j k}=$ efeito do k-ésimo animal dentro do i-ésimo grupo e do jésimo sexo $(\mathrm{k}=1, \ldots, 10) ; \mathrm{t}_{1}=$ efeito da l-ésima temperatura do ar $(1=20,30,40) ; b_{1} p_{1}=$ regressão sobre a pressão parcial de vapor; $b_{2} r_{1}=$ regressão sobre a taxa de evaporação total; $\mathrm{b}_{3} \mathrm{f}_{1}=$ regressão sobre a temperatura retal; $\mathrm{e}_{\mathrm{ijklm}}=$ resíduo.

Foram realizados contrastes ortogonais entre as três classes de temperatura, para cada variável dependente. Foi feita também a comparação entre as médias das variáveis fisiológicas medidas a 20, 30 e $40^{\circ} \mathrm{C}$, pelo teste de Tukey, a $5 \%$ de probabilidade, e estimados os coeficientes de correlação de Pearson entre as variáveis ambientais e fisiológicas medidas durante as três semanas para cada temperatura do ar, por intermédio do pacote estatístico SAS (1996).

\section{Resultados e Discussão}

Os índices obtidos na fase I, para cada animal, com o objetivo de selecioná-los em grupo 1 e grupo 2, estão apresentados na Tabela 1.

Na Tabela 2 é apresentado um resumo da análise de variância dos dados colhidos na fase II.

Para as três variáveis analisadas não houve diferença significativa entre os grupos (Tabela 2), sugerindo que os animais de ambos os grupos não apresentavam variação quanto à capacidade de adaptação às altas temperaturas ambientes. Houve diferença entre os sexos quanto à taxa de evaporação total, sendo essa de $2,14 \mathrm{~g} / \mathrm{kg} / \mathrm{h}$ para os machos e de $1,65 \mathrm{~g} / \mathrm{kg} / \mathrm{h}$ para as fêmeas. Tal diferença pode estar associada a menor frequiência respiratória apresentada pelas fêmeas $(175,5$ e 136,6 movimentos respiratórios/min. para machos e para fêmeas, respectivamente), apesar do sexo não ter apresentado efeito significativo na análise de variância para esta variável.

Os contrastes ortogonais entre as três classes de temperatura do ar, para cada uma das variáveis 
Tabela 1 - Índice de adaptação ao calor (ITF): obtido a partir da temperatura retal e da freqüência respiratória medidas na câmara climática durante a fase I e aplicado aos 21 ovinos para ordená-los em: grupo 1 (cinco animais mais adaptados ao estresse pelo calor) e grupo 2 (cinco animais menos adaptados ao estresse pelo calor)

Table 1 - Heat adaptation index (ITF): obtained considering the rectal temperature and respiratory frequency, measured in a climatic chamber during the first experiment and applied to the 21 sheep that were grouped in: group 1 (five animals more adapted to heat stress) and group 2 (five animals less adapted to heat stress)

\begin{tabular}{lc}
\hline Identificação dos animais & Índice (ITF) \\
Animals identification & Index (ITF) \\
\hline
\end{tabular}

\section{Fêmeas}

Females

\begin{tabular}{lc}
\hline 02 & 186.86847 \\
08 & 189.36850 \\
$12^{*}$ & 159.71862 \\
16 & 192.46853 \\
$18 \#$ & 203.86844 \\
$24 \#$ & 208.81871 \\
26 & 194.51872 \\
\hline Machos & Índice (ITF) \\
Males & Index (ITF) \\
\hline $01^{*}$ & 172.78469 \\
$03 *$ & 153.11386 \\
07 & 180.65850 \\
09 & 177.53830 \\
11 & 190.71775 \\
$13 *$ & 164.21631 \\
15 & 196.65703 \\
$17 \#$ & 215.03779 \\
$19 \#$ & 197.69461 \\
$21 \#$ & 219.21625 \\
23 & 191.84703 \\
27 & 181.15819 \\
$29 *$ & 164.09510 \\
31 & 190.71775 \\
\hline
\end{tabular}

* Média de ITF dos animais classificados como mais adaptados ao estresse pelo calor (Grupo 1).

\# Média de ITF dos animais classificados como menos adaptados ao estresse pelo calor (Grupo 2).

* ITF Means of animals classified as more adapted to heat stress (Group 1).

\# ITF Means of animals classified as less adapted to heat stress (Group 2).

(Tabela 2), demonstraram que houve diferença $(\mathrm{P}<0,01)$ quanto à taxa de evaporação nos animais, variando de 1,$49 ; 1,77$ e $2,43 \mathrm{~g} / \mathrm{kg} / \mathrm{h}$ para as temperaturas do ar a 20,30 e $40^{\circ} \mathrm{C}$, respectivamente. Para a frequiência respiratória, houve efeito $(\mathrm{P}<0,01)$ somente quando se comparou a temperatura do ar a $20^{\circ} \mathrm{C}$ com a de 30 e a de $40^{\circ} \mathrm{C}$, sendo que entre estas $\left(30\right.$ e $\left.40^{\circ} \mathrm{C}\right)$, não houve diferenças. Estes resultados concordam com aqueles obtidos por Titto et al. (1998), os quais observaram diferença significativa para frequiência respiratória entre ovinos submetidos a temperaturas máximas do ar abaixo e acima de $30^{\circ} \mathrm{C}$ $\left(28,5\right.$ e $\left.35,6^{\circ} \mathrm{C}\right)$.

De maneira geral, os ovinos responderam a uma carga térmica excessiva, conforme o esperado (Mendes et al., 1976; Baccari Jr., 1989; Silva \& Gaudiosi, 1995; Titto et al., 1998), com o aumento da freqüência respiratória, de tal forma que se elevou a dissipação do calor corporal por meio do aquecimento do ar inspirado e da evaporação nas vias respiratórias. Segundo Mendes et al. (1976), em ovinos, a freqüência respiratória pode atingir até 400 movimentos respiratórios por minuto.

Não houve efeito da temperatura do ar sobre a temperatura retal dos animais, o que permite inferir que houve manutenção da homeotermia, mesmo quando estes foram submetidos a $40^{\circ} \mathrm{C}$, demonstrando assim, a eficiência da termólise evaporativa no processo de termorregulação, o que está de acordo com os resultados de Titto et al. (1998). Estes resultados diferiram daqueles obtidos por Singh et al. (1980), que verificaram que, além da freqüência respiratória, a temperatura retal também aumentou, quando os ovinos foram submetidos a elevadas temperaturas do ar $\left(40\right.$ a $\left.45^{\circ} \mathrm{C}\right)$.

Constam na Tabela 3 as médias ajustadas das variáveis taxa de evaporação total, temperatura retal e frequiência respiratória e médias estimadas do calor dissipado por evaporação, para cada nível de temperatura do ar. $\mathrm{Na}$ Tabela 4 estão apresentados os coeficientes de correlação entre as variáveis ambientais e as variáveis fisiológicas medidas durante três semanas para cada temperatura.

A maior taxa de evaporação total obtida no presente trabalho $(2,43 \pm 0,15 \mathrm{~g} / \mathrm{kg} / \mathrm{h}$ - Tabela 3$)$ foi inferior àquela encontrada por Silva \& Gaudiosi (1995), de $3,99 \pm 0,12 \mathrm{~g} / \mathrm{kg} / \mathrm{h}$, e coerente com aquela de Hofmeyer et al. (1969), que relataram uma variação de 2,00 a 2,92 g/kg/h em ovinos da raça Corriedale, com aproximadamente dois anos de idade, mantidos a $38^{\circ} \mathrm{C}$.

Hofmeyer et al. (1969) verificaram também que o calor dissipado através da evaporação respiratória era responsável por cerca de $70 \%$ da evaporação total, sendo assim, 30\% ocorria por evaporação cutânea. Com base nesses dados, utilizaram-se os valores estimados para o calor dissipado por evaporação $(\mathrm{Q})$ e as médias da taxa evaporativa total 
Tabela 2 - Resumo da análise de variância da taxa de evaporação total, freqüência respiratória e temperatura retal, medidas nos dois grupos de ovinos submetidos a três níveis de temperaturas do ar

Table 2 - Summary of the analysis of variance of total evaporation rate, respiratory frequency and rectal temperature measured in two groups of the sheep under three levels of air temperatures

\begin{tabular}{|c|c|c|c|c|c|c|}
\hline \multirow[b]{2}{*}{$\begin{array}{l}\text { Fontes de variação } \\
\text { Sources of variation }\end{array}$} & \multicolumn{2}{|c|}{ TET } & \multicolumn{2}{|c|}{ FR } & \multicolumn{2}{|c|}{ TR } \\
\hline & $\begin{array}{l}\text { G } \\
D F\end{array}$ & $\begin{array}{l}\text { QM } \\
M S\end{array}$ & $\begin{array}{l}\text { G } \\
D F\end{array}$ & $\begin{array}{l}\text { QM } \\
M S\end{array}$ & $\begin{array}{l}\text { G } \\
D F\end{array}$ & $\begin{array}{l}\text { QM } \\
M S\end{array}$ \\
\hline Grupo & 1 & 0,042 & 1 & 695,881 & 1 & 0,868 \\
\hline $\begin{array}{l}\text { Group } \\
\text { Sexo } \\
\text { Sex }\end{array}$ & 1 & $1,492 *$ & 1 & 9397,878 & 1 & 3,311 \\
\hline $\begin{array}{l}\text { Animal dentro de grupo e sexo } \\
\text { Animal within group and sex }\end{array}$ & 5 & 0,126 & 5 & $2356,562^{*}$ & 5 & $1,358 * *$ \\
\hline $\begin{array}{l}\text { Temperatura do ar } \\
\text { Air temperature }\end{array}$ & 2 & $1,733^{* *}$ & 2 & $3612,705^{*}$ & 2 & 0,132 \\
\hline $\begin{array}{l}\text { Umidade do ar } \\
\text { Air humidity }\end{array}$ & 1 & $0,776^{*}$ & 1 & 579,045 & 1 & $0,311^{*}$ \\
\hline $\begin{array}{l}\text { Taxa de evaporação total } \\
\text { Total evaporation rate }\end{array}$ & - & - & 1 & 764,842 & 1 & 0,114 \\
\hline $\begin{array}{l}\text { Frequiência respiratória } \\
\text { Respiratory frequency }\end{array}$ & 1 & 0,150 & - & - & 1 & $0,386 * *$ \\
\hline $\begin{array}{l}\text { Temperatura retal } \\
\text { Rectal temperature }\end{array}$ & 1 & 0,371 & 1 & $6398,358 * *$ & - & - \\
\hline \multicolumn{7}{|l|}{$\begin{array}{l}\text { Contraste } \\
\text { Contrast }\end{array}$} \\
\hline $20^{\circ} \mathrm{C}$ vs $30^{\circ} \mathrm{C} ; 40^{\circ} \mathrm{C} 1$ & $1,17^{*}$ & 1 & $7132,267 * *$ & 1 & 0,000058 & \\
\hline $\begin{array}{l}30^{\circ} \mathrm{C} \text { vs } 40^{\circ} \mathrm{C} \\
\text { Resíduo }\end{array}$ & $\begin{array}{r}1 \\
57\end{array}$ & $\begin{array}{l}3,41 * * \\
0,186\end{array}$ & $\begin{array}{r}1 \\
57\end{array}$ & $\begin{array}{r}2708,655 \\
945,942\end{array}$ & $\begin{array}{r}1 \\
57\end{array}$ & $\begin{array}{l}0,184 \\
0,057\end{array}$ \\
\hline $\begin{array}{l}\text { Error } \\
\text { Total }\end{array}$ & 69 & & 69 & & 69 & \\
\hline $\begin{array}{l}\text { Total } \\
\text { Média geral } \\
\text { Overall mean }\end{array}$ & & 2,01 & & 165,29 & & 40,03 \\
\hline $\begin{array}{l}\text { Coeficiente de variação } \\
\text { Coefficient of variation }\end{array}$ & & 21,42 & & 18,61 & & 0,60 \\
\hline $\begin{array}{l}\text { Coeficiente de determinação } \\
\text { Coefficient of determination }\end{array}$ & & 0,34 & & 0,54 & & 0,81 \\
\hline
\end{tabular}

GL - Grau de liberdade (DF - Degree of freedom).

QM - Quadrados médios (MS - Mean squares).

** $P<0,01(P<.01)$.

* $P<0,05(P<.05)$.

(Tabela 3), obtendo-se os seguintes resultados: sob $\mathrm{TA}=20^{\circ} \mathrm{C}$, a termólise por evaporação respiratória (70\%) foi de $0,71 \mathrm{~W} / \mathrm{kg}$ e, aquela por evaporação cutânea $(30 \%)$ de $0,31 \mathrm{~W} / \mathrm{kg}$. Para $\mathrm{TA}=30^{\circ} \mathrm{C}$, os valores foram de 0,83 e $0,36 \mathrm{~W} / \mathrm{kg}$ para a evaporação respiratória e cutânea, respectivamente. Sob TA = $40^{\circ} \mathrm{C}$, foram obtidos os maiores valores para a evaporação respiratória e a cutânea, sendo esses: 1,13 e $0,49 \mathrm{~W} / \mathrm{kg}$, respectivamente.

Todos os valores foram menores que aqueles obtidos por Silva e Gaudiosi (1995), que encontraram 1,87 e $0,81 \mathrm{~W} / \mathrm{kg}$ para a evaporação respiratória e a evaporação cutânea, respectivamente, sob tempera- tura do ar de $35^{\circ} \mathrm{C}$ por duas horas. Porém, o resultado obtido para a evaporação respiratória $(0,83 \mathrm{~W} / \mathrm{kg})$ a $30^{\circ} \mathrm{C}$ foi semelhante aos de Brockway et al. (1965), que encontraram valor médio de $0,84 \mathrm{~W} / \mathrm{kg}$, sob a mesma temperatura em ovelhas e carneiros da raça Cheviot.

Para a taxa de evaporação total, entre os fatores considerados no modelo, a frequiência respiratória não teve efeito significativo $(\mathrm{P}<0,05)$ e a correlação entre a taxa de evaporação total e a frequiência respiratória mostrou-se baixa $(\mathrm{r}=0,236, \mathrm{P}<0,05)$ (Tabela 4), apesar de ser evidente que, sob altas temperaturas ambientes, o principal mecanismo de 
Tabela 3 - Médias ajustadas da taxa de evaporação total, da temperatura retal, da freqüência respiratória e do calor dissipado por evaporação medidos em ovinos Corriedale sob três temperaturas do ar $\left(20,30\right.$ e $\left.40^{\circ} \mathrm{C}\right)$

Table 3 - Adjusted means of total evaporation rate, rectal temperature, respiratory frequency and heat dissipated by evaporation, measured in Corriedale sheep under three levels of air temperatures $\left(20,30\right.$ and $\left.40^{\circ} \mathrm{C}\right)$

\begin{tabular}{lccc}
\hline Variável & $20^{\circ} \mathrm{C}$ & $30^{\circ} \mathrm{C}$ & \\
Variable & & & $40^{\circ} \mathrm{C}$ \\
\hline Taxa de evaporação total $(\mathrm{g} / \mathrm{kg} / \mathrm{h})$ & $1,49 \pm 0,18^{\mathrm{b}}$ & $1,77 \pm 0,11^{\mathrm{b}}$ & $2,43 \pm 0,15^{\mathrm{a}}$ \\
$\begin{array}{l}\text { Total evaporation rate }(\mathrm{g} / \mathrm{kg} / \mathrm{h}) \\
\text { Temperatura retal }\left({ }^{\circ} \mathrm{C}\right)\end{array}$ & $40,2 \pm 0,10^{\mathrm{a}}$ & $40,2 \pm 0,20^{\mathrm{a}}$ & $40,1 \pm 0,10^{\mathrm{a}}$ \\
$\begin{array}{l}\text { Rectal temperature }\left({ }^{\circ} \mathrm{C}\right) \\
\text { Freqüência respiratória }\left(\mathrm{min}^{-1}\right)\end{array}$ & $124,9 \pm 12,50^{\mathrm{b}}$ & $161,3 \pm 8,00^{\mathrm{a}}$ & $182,1 \pm 10,90^{\mathrm{a}}$ \\
$\begin{array}{l}\text { Respiratory frequency }\left(\mathrm{min}^{-1}\right) \\
\text { Calor dissipado por evaporação }(\mathrm{Watts} / \mathrm{kg})\end{array}$ & $1,02 \pm 0,38$ & $1,19 \pm 0,38$ & $1,62 \pm 0,30$ \\
Heat dissipated by evaporation $($ Watts $/ \mathrm{kg})$ & & &
\end{tabular}

Médias na mesma linha seguidas pela mesma letra diferem estatisticamente pelo teste Tukey $(P<0,05)$.

Means in the same row followed by the same letter statisticaly differ by Tukey test $(P<.05)$.

termólise em ovinos lanados é a evaporação respiratória (Brockway et al., 1965; Cena \& Monteith, 1975; Silva \& Gaudiosi, 1995; Silva, 2000). Tal suposição é reforçada pelo fato de que, na análise de variância da temperatura retal, a freqüência respiratória teve efeito significativo $(\mathrm{P}<0,01)$ sobre a manutenção da homeotermia dos ovinos (Tabela 2). Provavelmente, outros fatores fisiológicos estão envolvidos no processo de perda de calor de ovinos lanados para a manutenção do balanço térmico com o ambiente, porém esses não foram controlados no presente trabalho. Ademais, não houve correlação significativa entre a taxa de evaporação total e a pressão parcial de vapor $(r=0,185)$
$(\mathrm{P}>0,05)$, apesar da variação na pressão parcial de vapor (1,53 a 3,23 KPa), pois espera-se que quanto maior a umidade do ar, maior a tendência de afetar negativamente a evaporação no sistema respiratório. Entretanto, pode ter ocorrido uma compensação por meio do aumento da ventilação, como é demonstrado pelo coeficiente de correlação positivo $(\mathrm{r}=0,505)(\mathrm{P}<0,01)$ entre a pressão parcial de vapor e a freqüência respiratória (Tabela 4). Esse valor foi semelhante ao encontrado por Silva \& Gaudiosi (1995), $(\mathrm{r}=0,422)$ entre as mesmas variáveis, quando submeteram cordeiros das raças Ideal e Suffolk à temperatura do ar variando de 25 a $45^{\circ} \mathrm{C}$ e pressão parcial de vapor de 1,3 a $4,5 \mathrm{KPa}$.

Tabela 4 - Coeficientes de correlação entre as variáveis ambientais e as variáveis fisiológicas, medidas durante três semanas, para cada temperatura do ar

Table 4 - Correlation coefficients between environmental and phisiological variables measured during three weeks, for each air temperature

\begin{tabular}{|c|c|c|c|c|c|c|}
\hline & $\begin{array}{l}\mathrm{ET} \\
T E\end{array}$ & $\begin{array}{l}\mathrm{TR} \\
R T\end{array}$ & $\begin{array}{l}\text { FR } \\
R F\end{array}$ & $\begin{array}{l}\mathrm{Q} \\
Q\end{array}$ & $\begin{array}{l}\text { TA } \\
A T\end{array}$ & $\begin{array}{l}\mathrm{PV} \\
A H\end{array}$ \\
\hline Taxa de evaporação total (ET) & 1 & 0,135 & $0,236 *$ & 1 & $0,254 *$ & 0,185 \\
\hline $\begin{array}{l}\text { Total evaporation rate (TE) } \\
\text { Temperatura retal (TR) }\end{array}$ & & 1 & $0,293 * *$ & 0,129 & $0,306 * *$ & $0,297 * *$ \\
\hline $\begin{array}{l}\text { Rectal temperature }(R T) \\
\text { Freqüência respiratória (FR) }\end{array}$ & & & 1 & $0,221 *$ & $0,601 * *$ & $0,505 * *$ \\
\hline Respiratory frequency $(R F)$ & & & & & & \\
\hline $\begin{array}{l}\text { Calor dissipado por evaporação (Q) } \\
\text { Heat dissipated by evaporation }(Q)\end{array}$ & & & & 1 & $0,230^{*}$ & 0,165 \\
\hline $\begin{array}{l}\text { Temperatura do ar (TA) } \\
\text { Air temperature }(A T)\end{array}$ & & & & & 1 & $0,867 * *$ \\
\hline $\begin{array}{l}\text { Pressão parcial de vapor (PV) } \\
\text { Air humidity }(A H)\end{array}$ & & & & & & 1 \\
\hline
\end{tabular}




\section{Conclusões}

A utilização das variáveis fisiológicas: temperatura retal e frequiência respiratória, como principais parâmetros para avaliar a adaptação de ovinos ao clima tropical não é adequada. A correlação positiva e significativa encontrada entre a frequiência respiratória dos animais e a pressão parcial de vapor, demonstrou que há um aumento na ventilação respiratória com a elevação da umidade atmosférica, sendo que as trocas respiratórias constituem o principal mecanismo de termólise em ovinos lanados.

\section{Literatura Citada}

BACCARI Jr., F. Mecanismos adaptativos de ovinos lanados nos trópicos. In: SIMPÓSIO PAULISTA DE OVInOCUlturA, 1., 1989, Campinas. Anais... Campinas: Fundação Cargill, 1989. p.18-21.

BLIGH, J. The synchronous discharge of apocrine glands of the Welsh Mountain sheep. Nature, v.88, n.2, p.582-583, 1961.

BROCKWAY, J.M.; McDONALD, J.D.; PULLAR, J.D. Evaporative heat loss mechanisms in sheep. Journal Physiology, v.179, n.2, p.554-568, 1965.

CENA, K., MONTEITH, J.L. Transfer processes in animal coats. III. water vapour diffusion. Proceedings of the Royal of Society London B Biological Sciences, v.188, n.1, p.413-423, 1975.

FINCH, V.A. Body temperature in beef catle: its control and relevance to production in the tropics. Journal of Animal Science, v.62, n.1, p.531-542, 1986.

HALES, J.R.S. Physiology responses to heat. In: ROBERTSHAW, D. (Ed.) Environmental physiology. London: Buttherworths, 1974. 967p.

HOFMEYER, H.S.; GUIDRY, A.J.; WALTZ, F.A. Effects of temperature and wool lengh on surface and repiratory evaporative losses of sheep. Journal Applied Physiology, v.26, n.1, p.517-523, 1969.

HOLMES, C.R. Application of a weighing system for measuring total evaporative water losses in large ruminants. Journal Thermal Biology, v.10, n.1, p.5-7, 1985.
JOHNSON, K.G. Body temperatures and respiratory rates of free-ranging Merino sheep in and out shade during summer. Australian Journal Agricultural Research, v.42, p.13471357, 1991.

KOLB, E. Fisiologia veterinária. 2.ed. Zaragoza: Acribia, 1976. 1115p.

MENDES, M.A.; LEÃO, M.I.; SILVA, J.F.C. et al. Efeito da temperatura ambiente e do nível de energia da ração sobre os consumos de alimento e de água e algumas variáveis fisiológicas de ovinos. Revista da Sociedade Brasileira de Zootecnia, v.5, n.2, p.173-187, 1976.

MONTY, D.E.; KELLEY, L.M.; RICE, W.R. Acclimatization of St.-Croix, Karakul and Rambouillet sheep to intense and dry summer heat. Small Ruminant Research, v.4, p.379-392, p.1991.

SAS INSTITUTE. In: Procedures. versão 6, v.1. 3.ed. Cary: 1996.

SILVA, R.G. Introdução à bioclimatologia animal. 1.ed. São Paulo: Nobel, 2000. 286p.

SILVA, R.G.; GAUDIOSI, M.C. Termólise evaporativa em ovinos sob altas temperaturas. In: CONGRESSO BRASILEIRO DE BIOMETEOROLOGIA, 1., 1995, Jaboticabal. Anais... Jaboticabal: Legis Summa, 1995. p.193-203.

SINGH, M.; MORE, T.; RAI, A.K. Heat tolerance of different genetic groups of sheep exposed to elevated temperature conditions. Journal Agricultural Science, v.94, p.63-67, 1980.

SIQUEIRA, E.R. Estudo da produção, correlações fenotípicas e repetibilidade das características da lã em cinco raças de ovinos no sistema intensivo de pastejo. Jaboticabal: Universidade Estadual Paulista, 1990. 121p. Tese (Doutorado em Zootecnia) - Universidade Estadual Paulista, 1990.

TITTO, E.A.L.; NOGUEIRA FILHO, J.C.M.; VELLOSO, L. et al. Thermorregulation in sheep: rectal temperature, respiratory rate and water intake. Revista Portuguesa de Zootecnia, v.5, n.1, p.59-65, 1998. 